\title{
December 2019 Pulmonary Case of the Month: A 56-Year-Old Woman with Pneumonia
}

\section{Lewis J. Wesselius, MD}

Department of Pulmonary Medicine

Mayo Clinic Arizona

Scottsdale, AZ USA

\section{History of Present IIIness}

A 56-year-old woman complained of 6 weeks of increasing cough and shortness of breath. She had been treated for pneumonia with antibiotics, but when she failed to improve, she was begun on prednisone. She was receiving oxygen at $4 \mathrm{~L} / \mathrm{min}$ by nasal cannula at the time she was seen.

\section{$\mathrm{PMH}, \mathrm{SH}$, and $\mathrm{FH}$}

Her past medical history, social history and family were unremarkable other than a previous history of silicone breast implants. She was a nonsmoker.

\section{Physical Examination}

Her physical examination showed bibasilar crackles but was otherwise unremarkable.

\section{Radiography}

Her chest $\mathrm{x}$-ray is shown in Figure 1.

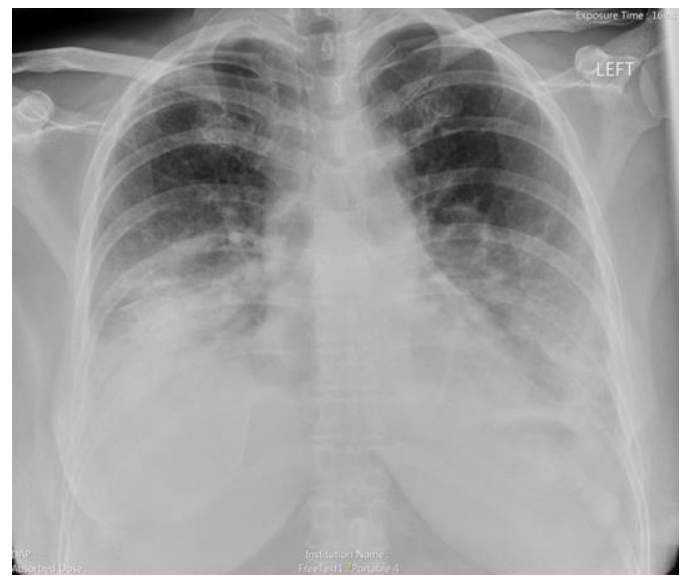

Figure 1. Patient's chest $\mathrm{x}$-ray taken 6 weeks after the beginning of her illness.

Which of the following should be done at this time?

1. Coccidioidomycosis serology

2. Sputum gram stain and culture

3. Thoracic CT scan

4. 1 and 3

5. All of the above 


\section{Correct! \\ 5. All of the above}

The chest x-ray shows bilateral consolidations. A thoracic CT scan is indicated to better define the consolidations. A coccidioidomycosis serology is nearly always indicated for any pneumonia in Arizona. Although sputum Gram stain and culture are generally not helpful in the initial diagnosis or treatment of community-acquired pneumonia especially those who have received antibiotics, most still order this in the work-up of a pneumonia (1).

Her Gram stain and sputum culture revealed only normal flora. Coccidioidomycosis serology showed the IgM within normal limits and an IgG was indeterminate. Her thoracic CT scan is shown in Figure 2.

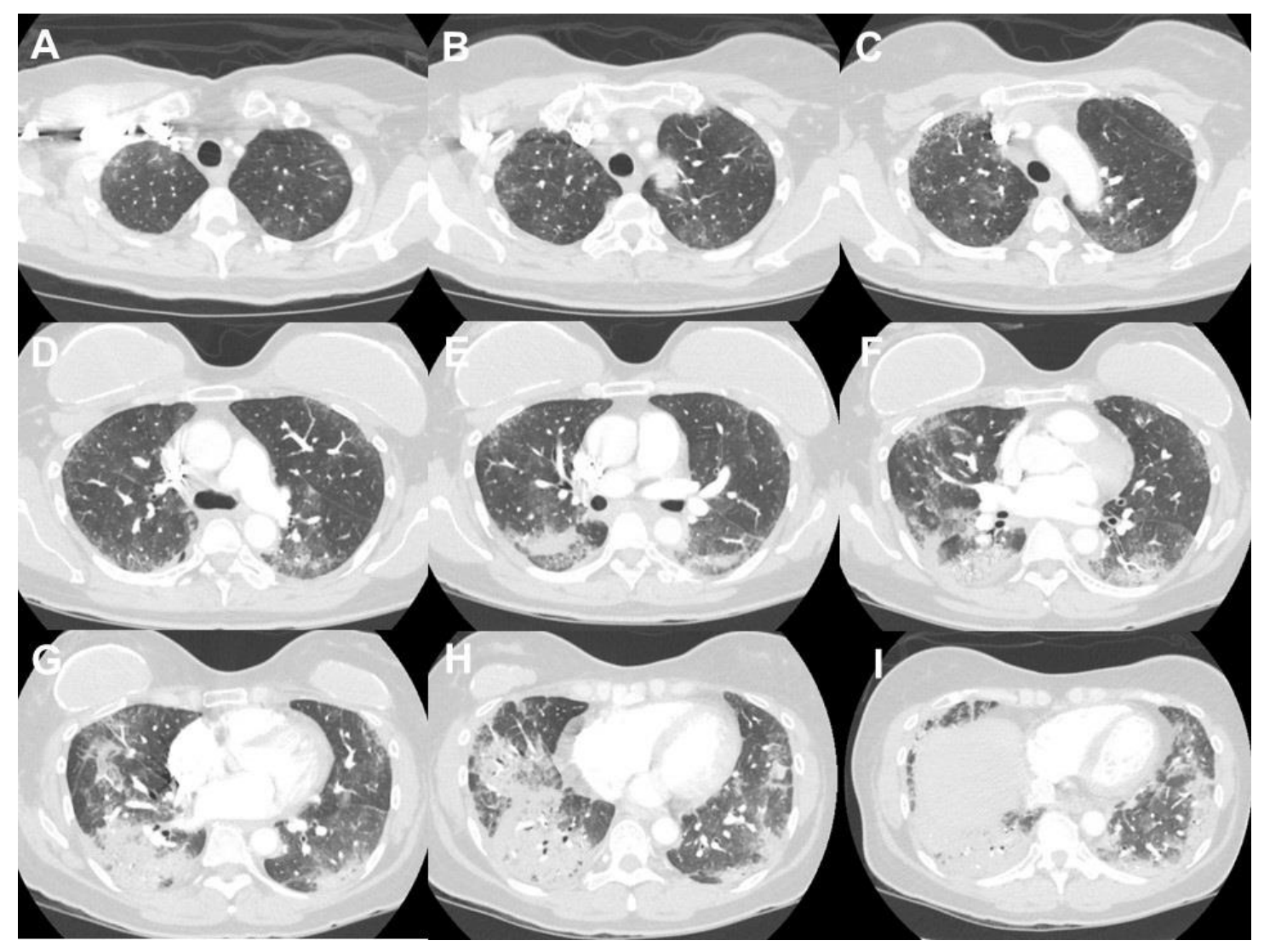

Figure 2. Representative images from the thoracic CT scan in lung windows.

Which of the following are considerations at this point?

1. Coccioidomycosis (Valley Fever)

2. Interstitial pneumonia

3. Silicone emboli

4. 1 and 3

5. All of the above 


\section{Correct! \\ 5. All of the above}

The thoracic CT scan demonstrates the bilateral consolidations in the lower lungs with a normal sized heart, no cavities, no effusions and an unremarkable mediastinum. The differential diagnosis is quite large. A not uncommon problem is the finding of a positive coccioidomycosis IgM or IgG assay where other serologic tests are negative (2). In such cases, the practitioner must interpret these results in the context of how likely, in the absence of serologic data, the clinical presentation is consistent with coccidioidomycosis. Leakage of silicone from her breast implants can cause a subacute pneumonitis (3). In addition to these considerations, interstitial pneumonitis from a rheumatologic disease represents a real consideration.

Which of the following should be done at this time?

1. Bronchoscopy with transbronchial biopsy

2. Fluconazole

3. Rheumatology consult

4. 1 and 3

5. All of the above 


\section{Correct!}

\section{1 and 3}

The next diagnostic step is probably bronchoscopy. Coccioidomycosis was thought to be unlikely although still within the diagnostic possibilities. Beginning fluconazole would not necessarily be wrong. A rheumatology consult was ordered and a number of serologic tests were ordered. Scl-70 and anti-nuclear antibody were positive, however, the consultant thought rheumatologic disease was unlikely. A bronchoscopy with transbronchial biopsy was performed. No organisms were seen on Gram, acid-fast, or silver stains. The biopsy was interpreted as showing "cryptogenic organizing pneumonia". Cryptogenic organizing pneumonia (COP), formerly known as bronchiolitis obliterans organizing pneumonia (BOOP), is an inflammation of the bronchioles (bronchiolitis) and surrounding tissue in the lungs (4). It should not be confused with bronchiolitis obliterans, a form of non-infectious pneumonia. It is often a complication of an existing chronic inflammatory disease such as rheumatoid arthritis, dermatomyositis, or it can be a side effect of certain medications such as amiodarone.

Which of the following should be done next?

1. Continue treatment with corticosteroids and follow-up

2. Repeat the bronchoscopy and transbronchial biopsy

3. VATS lung biopsy

4. 1 and 3

5. None of the above 


\section{Correct! \\ 3. VATS lung biopsy}

Cryptogenic organizing pneumonia is a nonspecific diagnosis (4). The patient was unsatisfied with the diagnosis and she had been treated with corticosteroids without improvement. In our experience a second bronchoscopy is usually no helpful if the first is nondiagnostic. For these reasons it was decided to proceed to a video-assisted thorascopic (VATS) biopsy. The histology is shown in Figure 3.

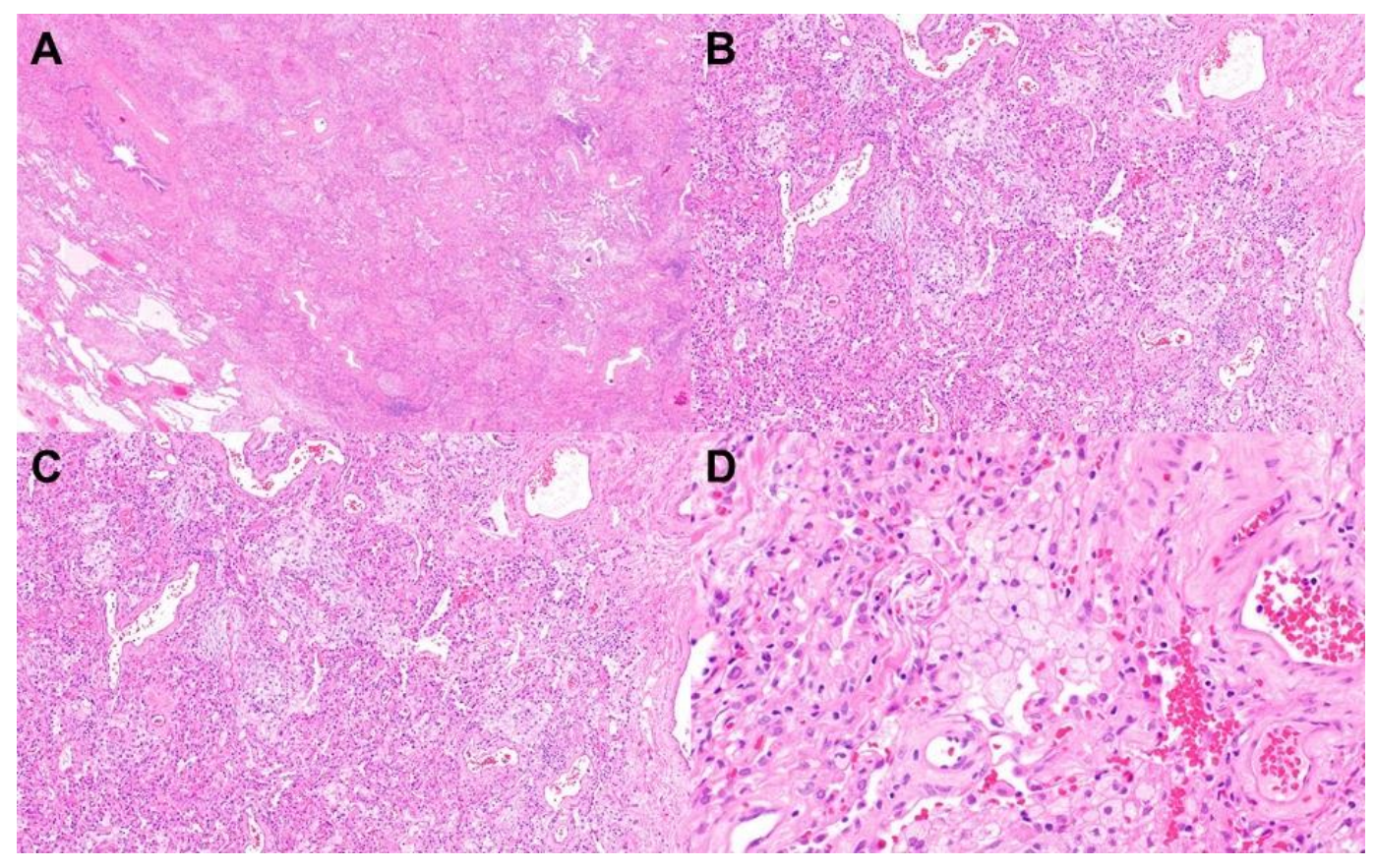

Figure 3. Representative H \& E microscopic images from the VATS lung biopsy at low $(A)$, intermediate $(B, C)$ and high power (D).

The biopsy was interpreted as showing acute lung injury, predominantly comprised of organizing diffuse alveolar damage and organizing pneumonia. There were collections of intra-alveolar foamy macrophages. No evidence of infection or silicone emboli were noted. Drug reaction, systemic connective tissue disorder, septicemia, noxious inhalants, sepsis/shock or in the idiopathic setting in acute interstitial pneumonia were listed as the most likely diagnosis.

Which of the following is the most likely diagnosis?

1. Acute interstitial pneumonia

2. Systemic connective tissue disorder

3. Vaping

4. 1 and 3

5. None of the above 


\section{Correct! \\ 1. Acute interstitial pneumonitis}

Vaping and an unidentified connective tissue disorder are possibilities. However, the patient denied vaping and there is no good evidence for a connective tissue disorder. Acute interstitial pneumonitis (AIP) was first described by Hamman and Rich in 1935 (5). This later became synonymous with idiopathic pulmonary fibrosis despite major clinical, radiologic and pathologic differences. Subsequently, the diagnosis was revisited and separated from idiopathic pulmonary fibrosis by Katzenstein et al. (6) in 1986. Diagnostic criteria for AIP are listed in Table 1.

Table 1. Diagnostic criteria for AIP.

- Acute LRT illness of less than 60 days

- Diffuse bilateral radiographic infiltrates

- Organizing or proliferative diffuse alveolar damage on lung biopsy

- Absence of any known inciting event or predisposing condition

- Absence of previously abnormal chest imaging

Which of the following represents the best course for further treatment?

1. Azathioprine

2. Continue with corticosteroids

3. Mycophenolate

4. Tacrolimus

5. The best course for treatment is not established 


\section{Correct! \\ 5. The best course for treatment is not established}

There is no established therapy for AIP. Of those listed, there is some evidence that mycophenolate might result in some improvement (6). She was begun on mycophenolate and showed some clinical improvement, no longer requiring oxygen at rest after 3 months. Her CT findings had also improved (Figure 4), but she still had restrictive changes on pulmonary function testing.

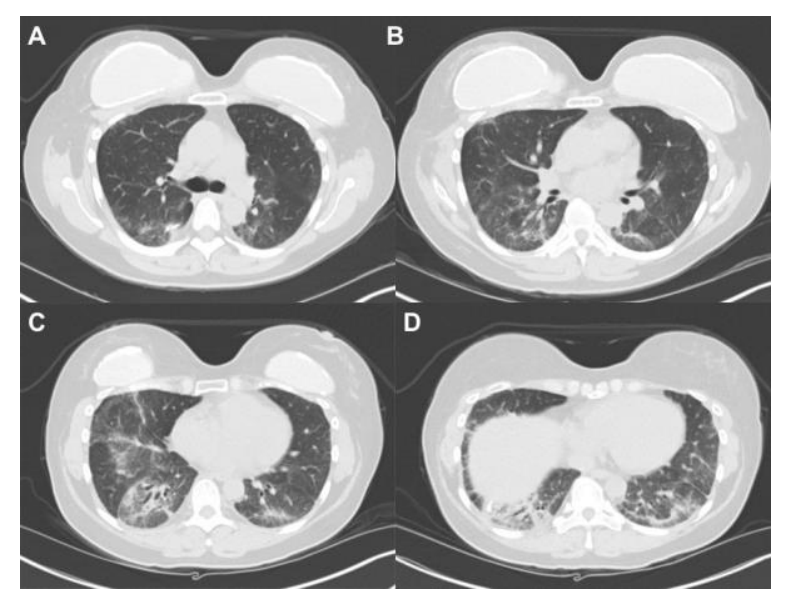

Figure 4. Representative images from repeat thoracic CT scan at 3 months follow-up.

\section{References}

1. Miyashita N, Shimizu H, Ouchi K, Kawasaki K, Kawai Y, Obase Y, Kobashi Y, Oka M. Assessment of the usefulness of sputum Gram stain and culture for diagnosis of community-acquired pneumonia requiring hospitalization. Med Sci Monit. 2008 Apr;14(4):CR171-6.

2. Ampel NM. New perspectives on coccidioidomycosis. Proc Am Thorac Soc. 2010 May;7(3):181-5. [CrossRef] [PubMed]

3. Ryu AJ, Glazebrook KN, Samreen N, Bauer PR, Yi ES, Ryu JH. Spectrum of chronic complications related to silicone leakage and migration. Am J Med. 2018 Nov;131(11):1383-6. [CrossRef] [PubMed]

4. Cordier JF. Cryptogenic organising pneumonia. Eur Respir J. 2006 Aug;28(2):42246. [CrossRef] [PubMed]

5. Vourlekis JS, Brown KK, Schwarz MI. Acute interstitial pneumonitis: current understanding regarding diagnosis, pathogenesis, and natural history. Semin Respir Crit Care Med. 2001 Aug;22(4):399-408. [CrossRef] [PubMed]

6. Katzenstein AL, Myers JL, Mazur MT. Acute interstitial pneumonia. A clinicopathologic, ultrastructural, and cell kinetic study. Am J Surg Pathol. 1986 Apr;10(4):256-67. [CrossRef] [PubMed]

7. McCoy SS, Mukadam Z, Meyer KC, Kanne JP, Meyer CA, Martin MD, Sampene E, Aesif SW, Rice LN, Bartels CM. Mycophenolate therapy in interstitial pneumonia with autoimmune features: a cohort study. Ther Clin Risk Manag. 2018 Nov 1;14:2171-81. [CrossRef] [PubMed] 